\title{
Pensamiento Gráfico y Diseño Colaborativo. Geometrías desarrollables en composiciones plegadas Arquitectónicas
}

\author{
Thinking Graphic and Design Collaborative. Developable geometries for folded architectural compositions
}

\author{
- Mauro Chiarella \\ UNL/CONICET, Argentina \\ chiarell@fadu.unl.edu.ar \\ - Andrés Martín-Pastor \\ USevilla, España \\ archiamp@us.es
}

\begin{abstract}
The design, manufacturing and assembly of SSFS (Same Slope - Folded Surface) Pavilion, is presented as a collaborative design experience recently developed between two Ibero-American universities (USevilla-UNL). In this experimental research, Graphic Thought feeds on the strategic use of developable geometries like to achieve outstanding manufacturing and installation of a temporary Folded composition. The practical exercise, results in a procedural model of open applications, nonlinear, for the generation of pavilions with different solutions adapted to each particular context.
\end{abstract}

Keywords: Temporary Architecture, Graphic Thought, CAD-CAM, Collaborative Design, Folded Compositions

\section{Introducción}

Desde la Universidad Nacional del Litoral (CID-FADU), Argentina, hemos desarrollado el estudio de las composiciones plegadas en arquitectura y diseño (propiedades espaciales y materiales) desde diversos enfoques y experiencias, que han dado como resultados líneas de investigación proyectual caracterizadas por la colaboración y presencia activa de grupos interdisciplinarios de diferentes universidades, a lo largo de más de una década de trabajo.

En el Departamento de Ingeniería Gráfica de la Universidad de Sevilla, nuestra experiencia de investigación se ha centrado en el uso de los fundamentos de Geometría Arquitectónica (Architectural Geometry), para abordar su vinculación prácticaalaprefabricacióndigitalylaconstrucción de soluciones reales en Arquitectura. Concretamente hemos investigado el desarrollo y la viabilidad de construcciones ligeras de arquitectura efímera en madera, la creación de sencillas leyes de generación geométrica parametrizadas, y su relación con las emergencias formales esas arquitecturas nos determinan. Este Proyecto compartido con la Universidad del Litoral debemos entenderlo como una parte de un proyecto de amplio recorrido y un proceso de investigación en Geometría Arquitectónica que iniciamos en 1999, que año tras año, se ha ido implementando.

De la alianza surgida entre ambas universidades, nace este proyecto interdisciplinar abierto, sustentado en la enseñanza de los fundamentos de geometría y fabricación digital, implementado desde el diseño colaborativo de un pabellón de madera capaz de adaptarse a las necesidades particulares de cada contexto.

\section{Retorno a los Fundamentos de Geometría}

Una de las ideas principales que ordena como un eje central todo el proyecto es la idea de la búsqueda/encuentro de un "fundamento geométrico" que permita, por sí mismo, explicar conceptualmente toda la obra arquitectónica. Ese fundamento geométrico lo hemos encontrado en la teorización y uso de las "superficies desarrollables", que aplicadas a la arquitectura, generan una familia de soluciones diversa y compleja y que no siempre ha estado explorada en todas sus variantes formales.

La razón del poco uso de la gran familia de superficies desarrollables en arquitectura, (ya que en la mayoría de los casos encontramos únicamente superficies cilíndricas y cónicas) tiene una explicación estrictamente académica. Esta razón podemos entenderla como una consecuencia de la extrema dificultad instrumental de ciertas operaciones gráficas al ser aplicadas a los métodos tradicionales de control de espacio, como los que se han usado de forma tradicional es la Geometría Descriptiva, con el sistema de Monge.

La teoría matemática y geométrica de las superficies desarrollables es sobradamente conocida. Así la encontramos 
en manuales de Geometría Analítica o Sintética. Sin embargo, en Geometría Descriptiva, donde se opera el estudio y control de la forma exclusivamente desde el pensamiento gráfico (y de forma tradicional en el Sistema de Monge) necesitamos producir en cada momento la doble proyección de la superficie o entidad geométrica objeto de estudio. El problema radica en que no siempre es operativo construir manualmente cada una de las representaciones cuando se sobrepasa cierto nivel de complejidad gráfica. Los límites operativos del Sistema de Monge, aparecerán junto a la incapacidad -o la lentitud- en generar una proyección.

De esta manera vemos que un conocimiento geométrico conocido y teorizado en matemáticas y en geometría analítica, no ha encontrado su mismo nivel de desarrollo en la Geometría Descriptiva Tradicional, la de Monge. De ahí que en los niveles formativos de la carrera de arquitecto, sólo nos hayan enseñado a "desarrollar" en el plano, figuras como el "cono" o el "cilindro", relegando al olvido, otras superficies igualmente desarrollables como son el Helicoide desarrollable", las convolutas desarrollables, las superficies de igual pendiente, las superficies tangenciales, las polares, etc.

\section{Pensamiento Gráfico Aumentado}

Nuestra reivindicación de volver a los fundamentos de Geometría desde el pensamiento Gráfico, viene apoyada por el uso de las nuevas tecnologías. Estas nuevas tecnologías CAD, tienen el problema de la representación ya resuelto desde un punto de vista instrumental. Los programas operan desde la propia geometría tridimensional, dando una amplia gama de recursos automatizados de control de la forma (de naturaleza geométrica) como la perpendicularidad, tangencia, ángulo, radios de curvatura, planos y círculos osculadores de curvas, etc. Esto hace que el problema geométrico en cuestión ocupe, desde el inicio, la máxima importancia, desplazando el problema de la representación a un segundo plano, casi eliminándolo por el concepto "construcción 3D". Se construye el propio cuerpo en un espacio tridimensional y las proyecciones bidimensionales aparecerán en la pantalla en movimiento, de forma automática y sin esfuerzo.

Esto no contradice a los objetivos de la Geometría Descriptiva como disciplina, sino que la lleva más allá de los límites conceptuales del sistema de Monge. A través del pensamiento gráfico (pensamiento gráfico aumentado por las nuevas tecnologías) se puede hacer una revisión de los fundamentos geométricos heredados, donde las cuestiones matemática-geométricas, pueden ser entendidas, asimiladas y resueltas exclusivamente desde lo gráfico. Nuestro proyecto de creación de un pabellón generado exclusivamente con "superficies desarrollables de igual pendiente", es un ejemplo de ello. Se trata de un problema que, concebido desde los fundamentos de Geometría que conocíamos a nivel teórico (Izquierdo Asensi, 1985; Gentil Baldrich, 1990) ha sido concebido y resuelto con razonamientos gráfico-geométricos: Concebir una superficie de igual pendiente que se apoya en diferentes arcos de elipses contenidos en el plano horizontal.

\section{Geometrías Desarrollables: Composiciones Plegadas}

Un tipo de geometría se define y clasifica a partir del estudio de las propiedades que permanecen invariantes en un objeto dado, mientras se le efectúan ciertas transformaciones. El número de invariantes como de transformaciones posibilitadas por cada geometría definen un estado particular de aproximación, comprensión o desarrollo en el proceso mismo de ideación. El desarrollo bidimensional de superficies tridimensionales plegadas posibilita repensar con creatividad métodos e instrumentos de ideación y fabricación. Los sistemas que permiten desarrollos automáticos de diferentes geometrías ponen en valor distintos significados desde el punto de vista expresivo y compositivo. Las geometrías de superficies desarrollables pertenecen a una tipología poco estudiada en la arquitectura y muy efectivas para la manufactura y montaje de composiciones plegadas. Las composiciones plegadas en arquitectura se pueden entender como un mecanismo de formulación proyectual y como una implementación constructiva. Se distinguen por su carácter de evolución formal, de transformación entre una superficie a un volumen con condiciones espaciales. Esta naturaleza de complejidad geométrica condiciona su aplicación, pero también expresa su potencialidad creativa. Avanzar desde la sugerencia de la manipulación de pliegues en papel hacia la definición geométrica y elaboración constructiva a escala real, es el desafío de estas composiciones. La composición plegada es a la vez una construcción formal, una acción operativa y una percepción sensible. Sus propiedades están vinculadas al volumen general y al tratamiento superficial que surge de una forma desdoblada, asumiendo un carácter funcionalperceptivo y material, implicando distintas consideraciones de enlace, comportamiento estructural y contención, que deben ser rigurosamente estudiadas para su adecuada ejecución.

\section{Proyectación y Materialidad Digital}

A este renacimiento de la Geometría Descriptiva, impulsada por los medios CAD, hay que añadir otro aporte instrumental de las nuevas tecnologías. Este aporte viene de la mano de la programación, no solo de la programación matemática, sino fundamentalmente de la programación de los propios razonamientos geométricos en una secuencia de pasos. Esto es lo que se lleva a cabo con programas como Grashopper que trabaja bajo Rhino, dando una dimensión todavía más profunda al problema del pensamiento gráfico aplicado al estudio de la Geometría. Esta dimensión nueva tiene que ver con la creatividad, ya no solo con el entendimiento de la naturaleza gráfica de un problema. La programación paramétrica ofrece no solo una solución a un problema, sino una familia entera de soluciones.

Teniendo en cuenta todo lo expuesto anteriormente: vemos que los fundamentos de geometría que se articulan 
al pensamientos gráfico con herramientas que aceleran la visualización (y por lo tanto la capacidad de síntesis), junto a la capacidad incluso de programar dichos pensamientos en cadenas de razonamientos más exigentes, que son capaces de buscar soluciones complejas dentro de una grandísima familia de soluciones, nos lleva a un nuevo paradigma, el paradigma de la nueva exploración de la forma arquitectónica.

Como sabemos, el diseño paramétrico introduce la geometría desde una visión matemático-algorítmica. Propone la generación de geometría a partir de la definición de una familia de parámetros iniciales y la programación de relaciones formales entre ellos. En estos procesos de diseño, la recurrencia a algoritmos y recursos computacionales avanzados no se utilizan simplemente para representar formas, sino para crear posibilidades proyectuales dinámicas y variables. El interés en la incorporación estratégica del concepto de diseño paramétrico al proceso de diseño radica en poder sumar nuevos recursos instrumentales que amplíen capacidades de respuesta en las disciplinas proyectuales. (Chiarella, 2012).

Los Sistemas CAD3D se ven multiplicados en su eficiencia gracias a su implementación con el software de diseño paramétrico (Grasshopper vía Rhino). Esto supone un gran avance en la investigación Geométrica al incorporar otro lenguaje de comunicación -la programación- al tradicional razonamiento gráfico asociado a la Geometría Descriptiva. Pero quizá lo más interesante es que, con esta herramienta, se articula el puente necesario para hermanar el aprendizaje de las matemáticas (geometría gráfica y analítica) con la programación, en un programa y/o proyecto unitario. De acuerdo con Pottmann (Pottmann, 2007), se trata de llenar el vacío existente entre las posibilidades técnicas y el conocimiento efectivo de los nuevos métodos de diseño geométrico.

Es así como la incorporación de la programación gráfica en la enseñanza del proyecto arquitectónico, no solo posee la potencialidad de desarrollar múltiples alternativas de diseño (mediante una formalización lógica del proceso, sus condiciones y relaciones geométricas), sino que además posibilitan integrarse con múltiples variables de análisis, aumentando la eficiencia del proyecto y su materialidad. El concepto Materialidad Digital propuesto por Gramazio \& Kohler, se sitúa en el complejo entretejido entre la programación computacional; la construcción o manufactura mediatizada; los datos y la materia, en las diferentes etapas del proyecto arquitectónico y su materialización. Parte de una provocación reflexiva hacia cierta inercia de la tradicional cultura arquitectónica, tanto en su expresión como en su capacidad productiva, estableciendo novedosos vínculos que amplían y enriquecen la relación entre la ideación, la tecnología y el entorno construído.

En nuestra experiencia, la delgada lamina multilaminada de madera guatambú (de 3 capas) de $6 \mathrm{~mm}$ de espesor cubre una distancia de aprox. 9mts mediante una deformación física aplicada por pliegue material y estudiada solo desde variables parametrizadas gráficamente al no contar con información técnica rigurosa del material utilizado. El resultado es una única envolvente continua autoportante y gran resistencia estructural que ha resistido hasta el momento ráfagas de viento de más de $50 \mathrm{~km} / \mathrm{h}$ (sudestadas y pamperos de la región Litoral Centro argentino). Las necesarias interacciones entre el modelo digital, los algoritmos de programación, la parametrización del diseño y las condicionantes de manufactura por control numérico han obligado a encontrar la máxima simplicidad en la idea generadora de la forma a fin de lograr que lo complejo sea la forma resultante y no la construcción y/o montaje de la misma.
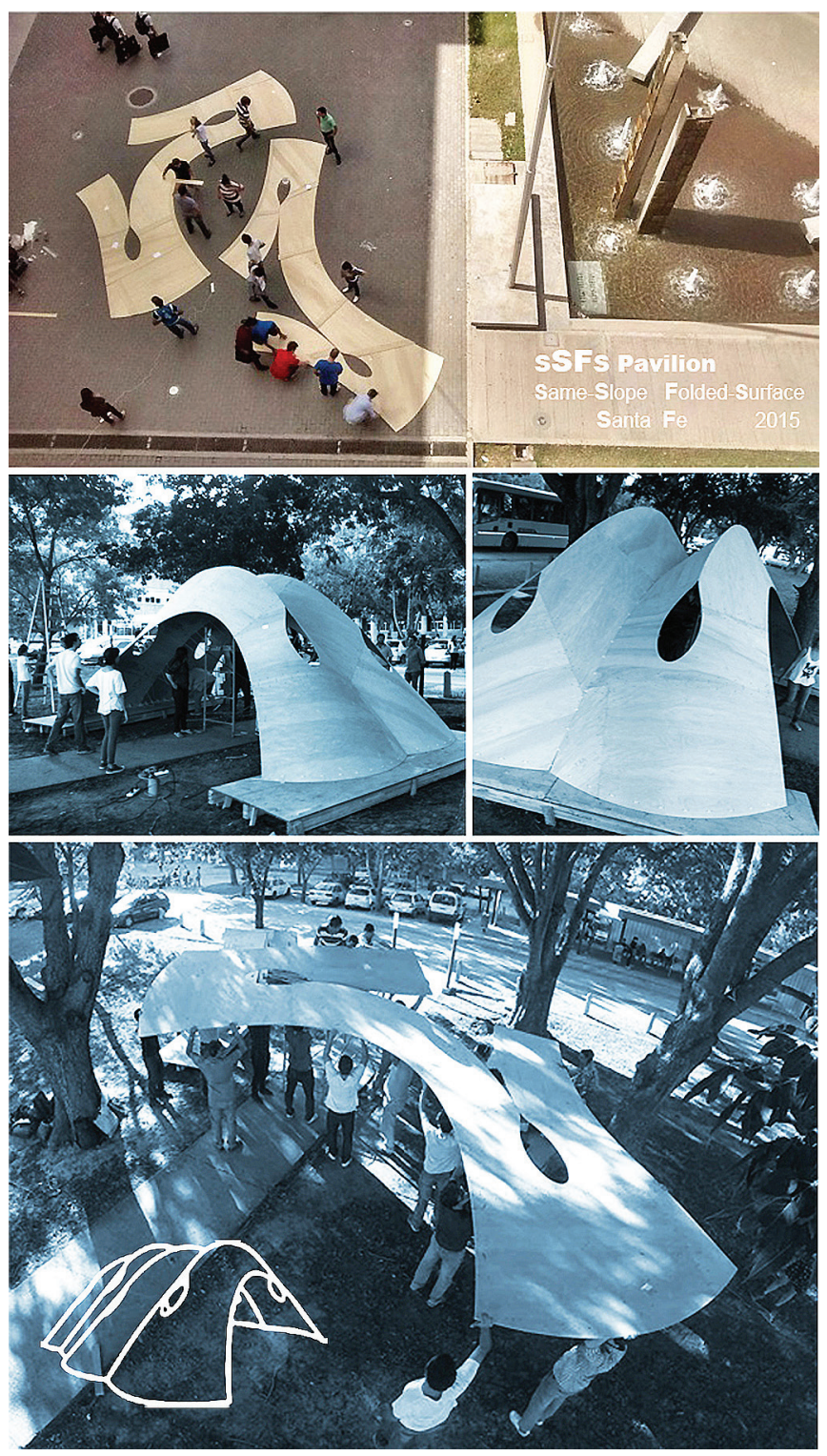

Figura 1: SSFS Pavilion. Proceso de montaje. Maestría en Arquitectura. Módulo: Proyectación y Construcción Digital. FADUUNL. Santa Fe. 2005. 


\section{Experiencia Didáctica de Diseño Colaborativo}

El conocimiento, desde la perspectiva psicosocial, no es un objeto que se pasa de un individuo a otro, sino algo que se construye por medio de operaciones y habilidades que se inducen en la interacción social. Es así como las situaciones de la enseñanza-aprendizaje no son invariablemente las mismas para diferentes sujetos, sino que dependen de un conjunto de condiciones complejas. Los instrumentos didácticos, por lo tanto, necesitan de máxima flexibilidad para adaptarse a éstas situaciones y no pueden sujetarse a la idea individual de quienes la utilizan. El conocimiento se construye en la interacción entre el individuo con una realidad compleja, múltiple y variable que supera cualquier pretensión simplificadora.

La sociedad de la información y las nuevas tecnologías potencian metodologías participativas y aprendizajes colaborativos. El aprendizaje colaborativo y el aprendizaje situado, coinciden en que todo aprendizaje tiene lugar en un contexto en el que los participantes negocian los significados. Así el aula se constituye en un campo de interacción de ideas, representaciones y valores en donde los alumnos, individualmente, obtienen diferentes interpretaciones de las mismas experiencias construyendo y reconstruyendo sus conocimientos según saberes, esquemas, y vivencias personales.

El principal cambio de paradigma en la acción docente es el abandono de la figura tradicional de transmisores de conocimiento para abordar el de conductores de los procesos propios de cada alumno acompañando la autogestión del conocimiento, su interrelación y puesta en práctica. De esta forma ya no es prioridad establecer solo una base de conocimiento en el individuo, en una memoria privada que uno debe llenarla con cosas que va usar en el futuro. Desde un concepto de memoria pública (bases de datos, bibliotecas de archivos, Internet) la información ya se encuentra disponible y asequible en tiempo real. Entonces lo que hay que enseñar es como llegar a ella, como usarla, como reciclarla. Se trata de privilegiar el análisis y la interpretación de la información sobre la acumulación de datos. Se prioriza la generación de conocimiento y la creatividad frente a las tradicionales técnicas de reproducción mnemotécnica. En el proceso de formación del proyectista, los nuevos medios plantean interrogantes hacia el tradicional modelo del proyecto de autor, proponiendo diferentes formas de colaboración ante el modelo romántico del proyectista único y solitario. El proceso de diseño en los nuevos medios implica trabajar con diferentes menús de varios programas, bases de datos de objetos digitales, bibliotecas de archivos y fundamentalmente trabajo en grupo conectado en red. Trabajar en equipo con flexibilidad y multiculturalidad es otra potencialidad creativa implícita en los nuevos instrumentos de aprendizaje.

$\mathrm{El}$ aprendizaje colaborativo es un método de enseñanza que utiliza la interacción social como medio para la construcción del conocimiento. Aquí la mayor parte de la responsabilidad de aprender está en los estudiantes. Ellos deben conceptualizar, organizar, poner a prueba las ideas, en un proceso continuo de evaluación y reconsideración de las mismas, asistidos por el profesor como facilitador de este proceso de enseñanza-aprendizaje.

La experiencia de SSFS (Same Slope - Folded Surface) Pavilion, ha sido una comprobación del uso del Diseño Colaborativo en dónde el Pensamiento Gráfico inicial (USevilla) se nutre de la utilización estratégica de geometrías desarrollables de igual pendiente para lograr la manufactura y montaje (UNL) de una composición plegada temporal. Iniciado en su diseño por el FabLab de la Universidad de Sevilla, puesto a prueba en dos de sus módulos por alumnos de FADU-UNL (Maestría en Arquitectura/Proyectación y Construcción Digital), el pabellón se construye en su totalidad en Sevilla en la Noche Europea de los investigadores el 25 de septiembre de 2015. Estas experiencias abiertas, las que no se construyen como una continuidad lineal, se realimentan de las reflexiones, análisis y producciones de cada etapa realizada.

En nuestra experiencia reciente, los diferentes grupos de alumnos FADU-UNL se han enfrentado, después de la experiencia de montaje de un diseño externo, a situaciones problemáticas a través de la interacción generada con los procesos de colaboración en el mismo sitio de trabajo y a través del intercambio de ideas con el diseño iniciado por GEOLAB (USevilla). Es así como a cada grupo se le ha solicitado que elabore, posteriormente al trabajo de montaje, una posible solución, mejora o valoración de la propuesta original de SSFS Pavillion. De esta forma se les asigna el compromiso de transferir un aporte constructivo en una mirada crítica y superadora de lo observado. La experiencia de taller colaborativo se realizó bajo la modalidad teórico-práctico, alternando video-conferencias y actividades presenciales de intercambio docente.

Como estrategia pedagógica propusimos desplazar el lugar de control de la clase de los profesores responsables a los grupos de alumnos, quienes definieron cuales eran los temas y problemas para la realización de su propio trabajo práctico, en relación a la experiencia recientemente aprendida. De esta forma los estudiantes se han podido presentar como co-constructores de su propio conocimiento más que consumidores de él. La complementariedad de información faltante o mejora de variable constructiva, de montaje o planificación del SSFS Pavilion a investigar, se ha definido y desarollado por los diferentes grupos desde los siguientes temas:

GRUPO 1: Propuesta o Estudio de Plataforma de apoyo que permita flexibilidad y transportabilidad en el montaje de pabellones efímeros (Diagnóstico: limitaciones contextuales observadas en las plataformas utilizadas).

GRUPO 2: Instructivo gráfico de Montaje de SSFS Pavilion (Diagnóstico: necesidad de lenguaje universal para la comunicación y coordinación de equipos).

GRUPO 3: Propuesta de materialidad (sobre la misma superficie envolvente) que posibilite el montaje con reducido 
números de operarios. (Diagnóstico: limitación en las estrategias de montaje)

GRUPO 4: Materialidades alternativas. Registro cualitativo y cuantitativo de opciones materiales. Comparaciones. (Variaciones proyectuales)

GRUPO 5: Construcción y armado de maqueta (escala 1:8) de SSFS Pavilion, con corte laser según piezas finales de manufactura CNC y montaje. (Objetivo didáctico)

GRUPO 6: Estudio de tiempos, recursos y organización de montaje para optimización del armado de SSFS Pavilion. (Diagnóstico: dispersión de tiempos y superposición de actividades en la experiencia SSFS-Santa Fe)

GRUPO 7: Propuesta de Materialidad Inflable para SSFS. (Variaciones proyectuales)

GRUPO 8: Propuesta de materialidad que considere permeabilidad visual con control de asoleamiento (patrones de corte). (Variaciones proyectuales sobre experiencia SSFS)

GRUPO 9: Propuesta de Materialidad metálica para SSFS. (Variación proyectual).
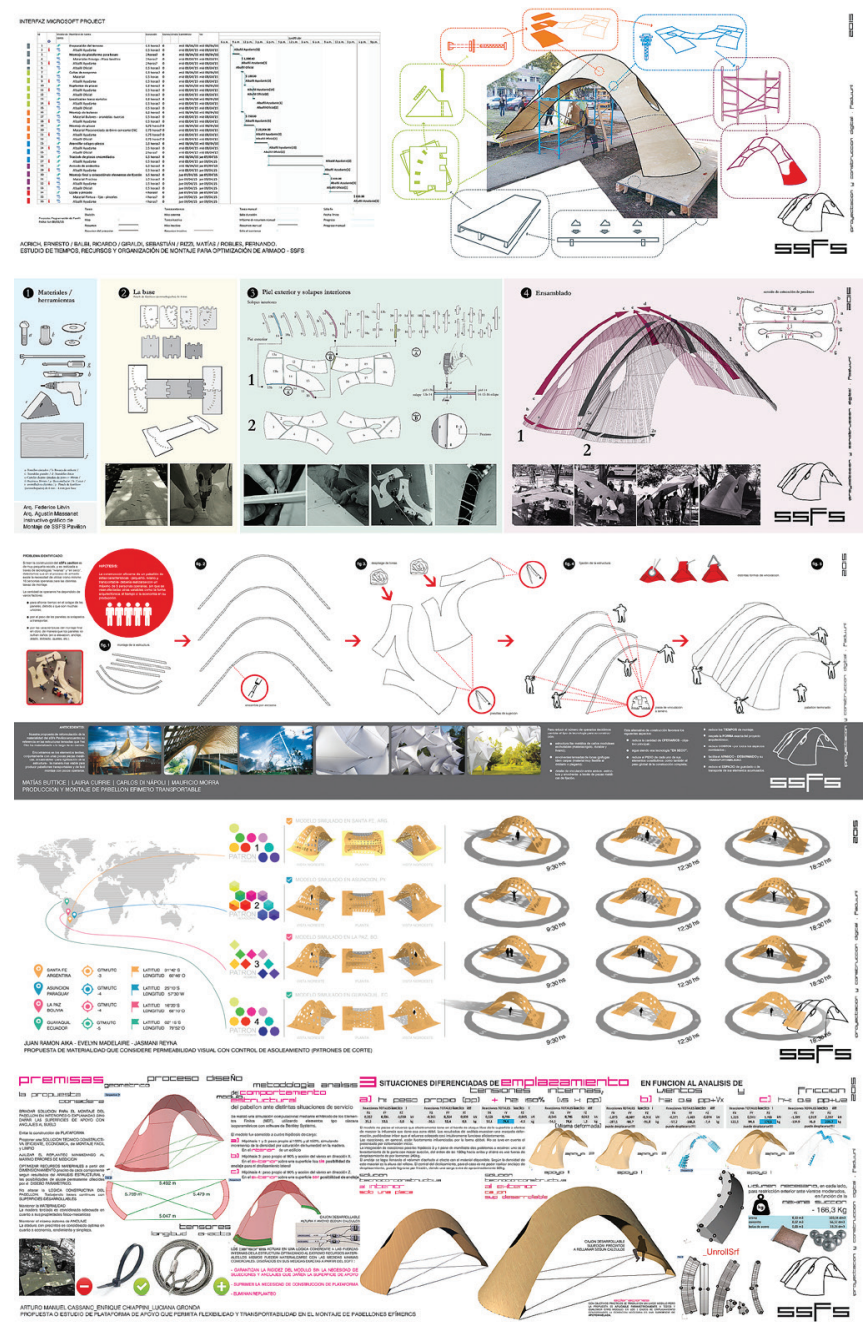

Figura 2: Maestría en Arquitectura 2015. Módulo: Proyectación y Construcción Digital. Desarrollos Grupo 6; 2; 3; 8 y 1.

\section{Conclusiones}

El éxito de un proceso de aprendizaje creativo se debería reflejar no sóloen la capacidad para comprender la complejidad del fenómeno de la arquitectura en toda su dimensión para actuar adecuada y flexiblemente en el manejo de situaciones concretas, sino también en el descubrimiento de nuevos instrumentos conceptuales y operacionales que amplíen las posibilidades mismas del pensamiento proyectual.

En relación a los múltiples instrumentos conceptuales y operativos con que cuenta la disciplina arquitectónica nos ha interesado trabajar sobre aquellas lógicas y procedimientos que no son habituales en la enseñanza tradicional de la misma y que derivan de una incorporación estratégica del Computational Making a los procesos creativos. En la contemporaneidad, la creatividad del sujeto se ha expandido significativamente en los procesos de intercambios e interacciones a través de la autoría colectiva, las bases de datos digitales y la construcción hipertextual de las tecnologías de la información y la comunicación. La propuesta de articular Pensamiento Gráfico y Diseño Colaborativo; Geometrías desarrollables y Composiciones plegadas tiene sus bases en esas premisas conceptuales.

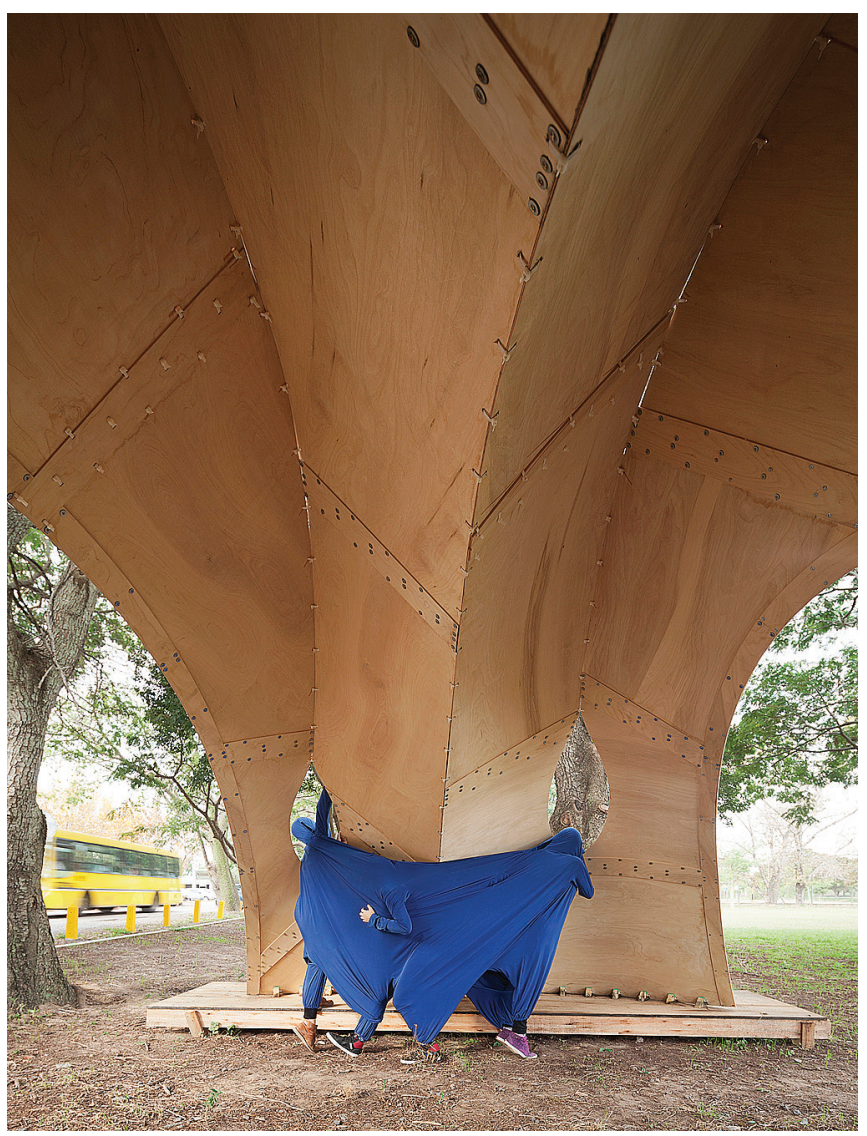

Figura 3: SSFS Pavilion. Acción "Nos somos pocos, es que estamos dispersos", Ariana Beilis. Colaboración: Carla Tortul, Felicita Cersofio. (https://vimeo.com/135685179) 
A nivel práctico, la experiencia de investigación y docencia conjunta ha impulsado nuestra arquitectura de diseño generativo, mejorando aspectos de prefabricación y avanzando en la fórmula que articulan los conocimientos de los Fundamentos de Geometría y la Prefabricación Digital. Concretamente ha supuesto un avance en la definición y cualificación de los prototipos arquitectónico que estamos desarrollando. Esto supone un avance en la construcción de sistemas constructivos y de montaje, más eficientes, ecológicos, sencillos de construir y de fácil puesta en obra.

Es así como hemos tenido presente la vinculación de los laboratorios de Fabricación Digital, FabLab, a la docencia e investigación de los fundamentos de Geometría para la Arquitectura. En nuestras propuestas hemos recogido el uso del FabLab, no solo como taller donde se dispone de unas maquinarias apropiadas para el corte del material, sino como algo más. El ejercicio de prefabricación en un taller de fabricación digital, es un simulacro a pequeña escala, de los entornos de los procesos productivos asociados a la construcción y la industria mediante la geometría gráfica informática. Por lo tanto casi de forma natural, es el escenario apropiado para llevar a cabo los trabajos de investigación propuestos desde un pensamiento gráfico aumentado.

\section{Créditos SSFS-Pavilion y Agradecimientos}

Maestría en Arquitectura FADU-UNL. Proyectación y Construcción Digital. Rodrigo García Alvarado/ Mauro Chiarella/ Andrés Martín Pastor.

Diseño y Proyecto: Dr. Andrés Martín-Pastor; Arq. Roberto Narváez-Rodríguez; Co-diseño paramétrico: Juan Expósito Bejarano. Dto. Ingeniería Grafica, Universidad de Sevilla. Equipo FABLAB Sevilla: José Pérez-de-Lama; Juan C. Pérez-Juidías.

Maestría en Arquitectura. FADU-UNL: Ernesto Vidal Acrich; Ricardo Gabriel Balbi; Lucia Barrado; Mariano Busaniche; María José Natalí Carreras; Paola Noé Colazo; Laura Gisela Currie; María Emilia Daveloza; Marjorie; Diaz Cruz; Carlos Andrés Dinapoli; Sebastián Alberto Giraldi; Adrián Gustavo Juri; Mauricio Esteban Morra; Jorge Luis Paggi Milans; Juan Pablo Ribeiro Collares; Matias Alejandro Rizzi; Fernando Gabriel Robles; Alberto Eduardo Zinno Vidal; Arturo Manuel Cassano; Enrique Luis Chiappini; María Luciana Gronda; Federico Litvin; Agustín Massanet; Juan
Ramón Aika Paiva; Andrés Francesconi; Guido Altuzarra; Mariano Benjamín Mendez Mena; Marianella Romina Katsini; Jasmani Lizandro Reyna Arguiño; Evelyn Noelia Madelaire Alarcon; Juan Pablo Temporelli. Plataforma: Arq. Paulo Chiarella; Germán Cristaldo; Nicolás Góngora y Sebastián Góngora. Fotografía audiovisual: Arq. Federico Cairoli. Acción "No somos pocos, es que estamos dispersos" Ariana Beilis. Colaboración: Carla Tortul, Felicita Cersofio.

\section{Referencias}

Chiarella, M.; García Alvarado, R. (2014). Folded Compositions in Architecture: Spatial Properties and Materials. Nexus Network Journal. Springer. DOI: 10.1007/s00004-014-0226-4 Chiarella, M. (2012). Folded Geometries in Architecture. Parametric Design and Digital Manufacturing. The 9th international, interdisciplinary Nexus conference for architecture and mathematics: Relationships Between Architecture and Mathematics. Milan, Italy.

Gentil Baldrich, J. M. (1990). Método y aplicación de representación acotada. Ed. Cuadernos EGA. Universidad de Sevilla. España.

Izquierdo Asensi, F. (1985). Geometría descriptiva superior y aplicada. Editorial Dossat. Madrid. España (pag. 308)

Martin-Pastor, A; Torres-Holguín, J; Narváez-Rodríguez, R; Galindo-Diaz, J. Los workshops de geometría en Cad3d y prefabricación digital como estrategia docente en la enseñanza de la geometría para la arquitectura. Geometría y proyecto" p. 213-216. In: Proceedings of the XVIII Conference of the Iberoamerican Society of Digital Graphics: Design in Freedom. Montevideo. 2014.

Narváez-Rodríguez, R; Martín-Pastor, A; Aguilar-Alejandre, M. (2014). The Caterpillar Gallery: Quadratic Surface Theorems, Parametric Design and Digital Fabrication. Advances in Architectural Geometry 2014-2015 (Editors: P. Block; J. Knippers; N. J. Mitra; W. Wang).

Pottmann H, Asperl A, Hofer M and Kilian A. (2007). Architectural Geometry. Bentley Institute Press.

Narváez-Rodriguez, R; Martín-Pastor, A; Infante-Perea, M; Aguilar-Alexandre, M. (2014). Teaching Innovation in Architectural Geometry; Fundamentals, Digital Fabrication and Collaborative Work. Proceeding of XII Graphic Expresion Applied to Building International Conference. New Technics, same Fundaments. pp. 94-103. Editorial Rueda, Madrid 2014. 